medicine and paediatrics, and I am now a lecturer and senior registrar in paediatric oncology. There's nothing unusual about this, except for the fact that I suffer from severe bilateral sensorineural hearing loss of 70-80 dB and need to use postauricular hearing aids and an electronically amplified stethoscope.

When I attended a careers meeting at the age of 16, I was advised by a lecturer in social medicine that I had no chance of a medical career. My "deafness" itself seemed to be reason enough - she made no attempt to discus its severity or inquire how I managed at school. Undaunted, my father and I wrote to the admissions tutors of eight medical schools. The favourable replies from Birmingham and Newcastle encouraged me to apply. After normal interviews, both medical schools offered me a place. I trained at Birmingham, and now find myself in Newcastle.

Many other people told me that I should (not could, an important distinction) forget about wanting to be a doctor. They had the best of intentions-wishing to protect me from the likelihood of disappointment. However, I had wanted to be a doctor since I was six years old and that was all there was to it. Once I was at medical school, my ENT consultant and others suggested various non-clinical careers or "less taxing" clinical careers. But by the time I decided to train in paediatrics they knew me well enough not to attempt to dissuade me.

I do not think that I have needed more natural ability or intelligence than the average medical student. What I have needed is determination, and support from my family, friends, colleagues, teachers, and patients. Every medical student and doctor needs these to a greater or lesser degree.
I do not feel that my hearing loss has had any effect whatsoever on my medical career so far, and I am hopeful that it will not do so in the future. Colleagues, teachers, and patients have treated me as a normal doctor, which is how I see myself. Many close friends and colleagues say that they never think of me as being deaf.

I do not want to paint too rosy a picture of life as a doctor with hearing loss. My ENT surgeon believed that I would always have to work much harder than others at hearing and understanding, and that this would prove very taxing - I have no way of comparing, but I suspect that he was probably right.

However, being deaf and wearing hearing aids has some compensations (I appreciate the peace and quiet of night when I take my aids out) and lighter moments: as a student, I learnt that the whistle due to acoustic feedback from hearing aids can mimic bleeps, epecially "cardiac arrest" calls, perfectly.

\section{An insurmountable barrier?}

What is our conclusion? We believe that deafness, or any other chronic illness or disability, is not necessarily an insurmountable barrier to a medical career. Only when the person is considered as a whole can decisions on his or her suitability for a job or degree course be made. Evaluation of how disabled applicants cope with daily life and school may be very informative - if they have managed to get as far as seriously considering their chosen career, they may have much to offer it.

1 Walters S. A doctor despite a disability. BM 1988;297:1665-6.

\title{
The practical physics of hearing aids
}

\author{
N D N Belham
}

Audiometric testing shows that, with the onset of old age, the general sensitivity of hearing decreases and the frequency response of the ear falls off rapidly for sounds of high pitch - above about $2000 \mathrm{~Hz}$. The basic electronic hearing aid consists of microphone, amplifier, and earpiece, so it would seem logical to increase the amplification for high pitched sounds. Unfortunately this action has an unwanted side effect, which can be severe enough to cause some people to discard the aid altogether: it enhances room noise considerably. The results of several simple experiments show how hearing aids, and the conditions for their use, can be improved.

\section{Basic improvements}

Journalists are often shown thrusting their microphones quite close to the face of the interviewee in order to cut down unwanted sound. Pop stars act in a similar manner, almost swallowing their microphones to cut down feedback from the blasting loudspeakers. This suggests an answer to the problem of amplification experienced by hearing aid users. It is to connect the microphone to a long lead and to place it near to the source of the wanted sound. Electret microphones are most suited to be placed near a television as they are not affected by the magnetic fields produced there. Several friends were fitted up in this way - to the satisfaction of both patient and spouse. The technique was extended when a group of people visited a person with hearing difficulties. For the experiment a ring main was fitted round the edge of the room with a plug box near each chair and the television. Small microphones were clipped to the clothing of each person, rather like on a chat show. At the end of the afternoon the daughter remarked that she had not heard her father talk so much for years.

A very simple amplifier is quite effective. A low noise transistor (for example, BC109) followed by a low power integrated circuit, such as the LM386, will do the job. The cost of the components, including $8.4 \mathrm{~V}$ PP3 nicad rechargeable battery and lightweight headphones, need not exceed $£ 20$.

\section{Hearing on the move}

Experiments were then conducted to test ways of dispensing with the microphone lead. A pair of walkietalkies is a possible solution but has two disadvantages. The first is lack of privacy. The radio signal will travel all over the neighbourhood and anyone with a suitable receiver can pick it up. Chance remarks could therefore lead to social chaos. The second disadvantage is that such a system is not adaptable for group use. More than one transmitter tuned to close frequencies will produce audible heterodyne tones (whistles).

Another possibility is to use infrared as a link. Although this worked, daylight produced a continuous background hiss and artificial light produced a steady hum owing to current alternations. To overcome these two disadvantages powerful transmitters would be needed. This system would be a heavy drain on battery power as hearing aids are used for long periods.

Magnetic loops are very costly compared with a
N D N Belham, retired

physics teacher

BMF 1992;305: 1569-70 


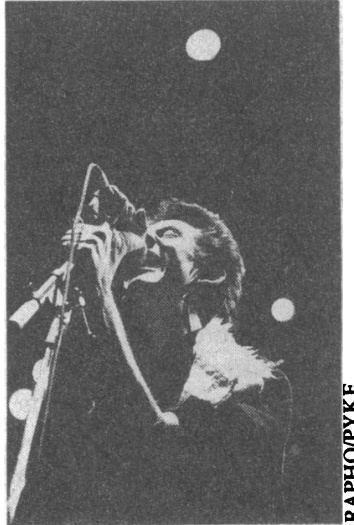

Almost swallowing the microphone cuts down feedback from blasting loudspeakers microphone lead and become rather complicated when used in a group situation.

\section{Room noise}

The cause of the enhancement of room noise called for further thought. Part of the problem is the multiple reflection of sound from the surfaces of the room and its contents. High pitched sounds behave differently from those of low pitch. This is because the wavelength of high pitched sounds is only a few centimetres while that of low pitched sounds is a few metres. Waves tend to "swirl" around hard objects that are small compared with the wavelength but are reflected by objects larger than the wavelength. In most rooms there are more hard surfaces and objects measured in centimetres than metres, so high pitched sounds are reflected many more times than those of low pitch. The fading reflections (reverberations) of any sound therefore have many more high frequency components than low. Processed by a hearing aid, this often produces an unpleasant shrillness. Sharp sounds, such as cutlery being placed on a table, can be devastating. The reflections reach the listeners at different times, resulting in a lack of clarity. These effects are increased if the hearing aid is designed to boost the higher frequencies.

\section{Feedback}

Feedback can also cause problems. Electrical feedback is produced when energy from the output end of the amplifier reaches the input. This usually happens via the supply line and can be cured by careful design. Such feedback can take the form of a chugging sound ("motor boating") or a sustained audio tone, either of which drowns the wanted speech, or an oscillation too high to be audible but which "chokes" the amplifier. The increase in internal resistance of a failing battery can act as a coupling element between parts of the circuit and also cause electrical feedback.

The familiar sustained acoustic feedback is brought about by sound from the earpiece reaching the microphone in sufficient intensity and correct phase to produce a steady tone. Unfortunately the pitch of this tone can be so high that the wearer does not hear it but, knowing something is wrong, turns up the volume! The frequency of the steady tone $(4000 \mathrm{~Hz})$ turned out to be the resonant frequency of the earpiece. The sound output is much enhanced at resonance but can be reduced to the general level by inserting a narrow stop band filter in the amplifier circuit. The general volume can then be turned up considerably before acoustic feedback again becomes noticeable.

Feedback that is insufficient to create a sustained tone can still enhance the amplification of frequencies near that tone. In practice this usually happens with high pitched sounds. The "over the ear" type of aid is particularly prone to feedback since the microphone is only a few centimetres from the exit of the tube carrying the sound. To prevent feedback the ear plug needs to be a good fit, but it also must allow pressure equalisation in the medium term-it must be fitted with as much care as a denture.

\section{Clarity and filters}

In the group experiment previously described one man had an exceptionally deep and "gravelly" voice which the partially deaf person could not hear well enough to understand. This problem was made the subject of further experiments. By using a recording of the voice, its general pitch was raised with a processing chip designed for use in talking toys. As a result the clarity was improved, but the high pitched components causing the gravelly effect remained.

Attention was then turned to the use of electronic filters. It was found that intelligent speech could be transmitted using a much narrower band of frequencies than usually provided by hearing aid manufacturers. When such a filter was constructed it was found that the gravelly voice was much improved, and-more importantly-it made the speech "stand out" from the reverberation. The filter reduced those components of the reverberation that fell outside its pass band.

More filters were constructed with centre pass frequencies of $1200 \mathrm{~Hz}, 1000 \mathrm{~Hz}, 845 \mathrm{~Hz}$, and $500 \mathrm{~Hz}$. In each case the width of the $6 \mathrm{~dB}$ (half the peak value) band was a little under 2.5 octaves. The best compromise seemed to be the filter centred at $845 \mathrm{~Hz}$. The loss of the lower frequencies did not reduce intelligibility but certainly reduced room noises. The loss of the high frequencies also reduced room noises, and particularly the shrillness, and made little difference to intelligibility.

The loss of the lower frequencies has other advantages. The ear has some power of automatic volume control, which is disabled by large amplitude sounds. In general, low pitch sounds have relatively larger amplitudes than those of higher frequencies and so are more likely to bring about this effect. In elderly people concentration is essential and any sound unrelated to intelligibility is a disadvantage. The ear is a non-linear device and produces harmonics of lower frequencies, which can interfere with perception of higher frequencies.

The filter, as well as cutting down room noise, helps when used with a group of people. One 85 year old wrote in thanks that she found the aid very beneficial when talking with the family.

There is much to be said for the box type of aid for elderly people. For one thing it allows full size controls to be used, thus easing the problems of arthritic fingers.

It is hoped that this short note will help readers to appreciate the physics behind some difficulties commonly experienced by patients wearing the less sophisticated hearing aids, and also that manufacturers will be encouraged to incorporate band pass filters in future aids. The filter experiments would suggest that the principle of hearing aid design should be changed - from compensating for loss of hearing in the top range, to putting the maximum energy into the band surrounding the frequency $(1000 \mathrm{~Hz})$ where the ear is most sensitive.

\section{Useful addresses}

A list of publications is available from Royal National Institute for Deaf People, 105 Gower Street, London WC1E 6AH (tel 0713878033 ).

Electronic components and massive catalogues containing much detail are available from:

Maplin Electronics, PO Box 3, Rayleigh, Essex SS6 8LR (tel 0702 554161)

Cirkit Distribution Ltd, Park Lane, Broxbourne, Hertfordshire EN10 7NQ (tel 0992 444111).

For those who may wish to experiment, circuit diagrams can be supplied by the author on receipt of a stamped, addressed envelope.

1 Hearing, Deafness and Electronic Technology. Electronics-The Maplin Magazine. 1991; No 42,43,44.

2 Terman FE Radio engineering. New York: McGraw-Hill, 1955. 